\title{
Synthesis, Optical Characterization, and Size Distribution Determination by Curve Resolution Methods of Water-Soluble CdSe Quantum Dots
}

\author{
Calink Indiara do Livramento Santos ${ }^{a}$, Melissa Souza Carvalho ${ }^{a}$ Ellen Raphael ${ }^{a}$ Clecio Dantas ${ }^{b}$ \\ Jefferson Luis Ferrari", Marco Antônio Schiavon ${ }^{a *}$ \\ ${ }^{a}$ Grupo de Pesquisa em Química de Materiais - GPQM, Departamento de Ciências Naturais, \\ Universidade Federal de São João del-Rei-UFSJ, Campus Dom Bosco, Praça Dom Helvécio, 74, CEP \\ 36301-160, São João del-Rei, MG, Brazil \\ ${ }^{b}$ Laboratório de Química Computacional Inorgânica e Quimiometria - LQCINMETRIA, Departamento \\ de Química e Biologia, Universidade Estadual do Maranhão - UEMA, Campus Caxias, Praça Duque \\ de Caxias, s/n, CEP 65604-380, Caxias, MA, Brasil
}

Received: February 14, 2016; Revised: July 6, 2016; Accepted: September 28, 2016

In this work a colloidal approach to synthesize water-soluble CdSe quantum dots (QDs) bearing a surface ligand, such as thioglycolic acid (TGA), 3-mercaptopropionic acid (MPA), glutathione (GSH), or thioglycerol (TGH) was applied. The synthesized material was characterized by X-ray diffraction (XRD), Fourier-transform infrared spectroscopy (FT-IR), UV-visible spectroscopy (UV-Vis), and fluorescence spectroscopy (PL). Additionally, a comparative study of the optical properties of different CdSe QDs was performed, demonstrating how the surface ligand affected crystal growth. The particles sizes were calculated from a polynomial function that correlates the particle size with the maximum fluorescence position. Curve resolution methods (EFA and MCR-ALS) were employed to decompose a series of fluorescence spectra to investigate the CdSe QDs size distribution and determine the number of fraction with different particle size. The results for the MPA-capped CdSe sample showed only two main fraction with different particle sizes with maximum emission at 642 and $686 \mathrm{~nm}$. The calculated diameters from these maximum emission were, respectively, 2.74 and $3.05 \mathrm{~nm}$.

Keywords: Semiconductors, Optical materials, CdSe, Optical properties, size distribution

\section{Introduction}

Colloidal semiconducting nanocrystals, also known as quantum dots (QDs), have attracted widespread attention over the last decade-QDs display novel optical properties such as intense luminescence, narrow and symmetric emission, broad and strong absorption, and high stability in presence of photo and chemical radiation. ${ }^{1}$ QDs are particularly interesting for fundamental studies in the field of nanoscience, have potential technological applications in optoelectronic devices, including light emitting diodes (LEDs), lasers, photodetectors, and photovoltaic cells, ${ }^{2-5}$ and may aid labeling of biomedical entities. ${ }^{6}$ Quantum confinement effects give rise to semiconducting nanoparticles with physical and chemical properties that are distinct from the properties of the individual molecule or the bulk species. ${ }^{7}$ The possibility of confining charge carriers (electrons and holes) in three dimensions endows these intrinsic semiconductors with a size-dependent band gap and with optical properties that strongly rely on nanoparticle size and structure. ${ }^{8,9}$ Kamat et al. ${ }^{10}$ reported on the specific advantages of using QDs as light harvesting assemblies in solar cells. The size effect allowed these authors to tune the visible response, vary the band offsets, and modulate the vectorial charge transfer

* e-mail: schiavon@ufsj.edu.br across particles of different sizes. This effect could pave the way for using hot electrons or generating multiple charge carriers with a single photon.

Cadmium selenide (CdSe) is one of the most important materials among the various kinds of II-VI semiconductors. Its size-tunable emission in the visible range has made it a useful model system to investigate a wide array of electronic, optoeletronic, and chemical processes at nanoscale. ${ }^{11} \mathrm{CdSe}$ QDs display a direct band gap of $1.74 \mathrm{eV}$, which makes their use in solar cells and other electronic devices feasible. ${ }^{10-14}$ Reactions in organic media constitute the main route to obtain CdSe QDs. ${ }^{15-17}$ These synthetic approaches produce highquality optical nanoparticles ${ }^{18}$ with narrow size distribution. ${ }^{17,19}$

The preparation of CdSe QDs in aqueous media is reproducible and inexpensive, and the resulting water-soluble QDs present low toxicity in comparison with that produced in organic media. ${ }^{11,20,21}$ Because the large absorption and emission bands of water-soluble CdSe nanocrystals remain poorly understood, there is little information about their size distribution. Exchange of the short-chain surface ligand for a long-chain ligand renders water-soluble CdSe QDs easily soluble in organic solvents, ${ }^{22}$ as shown by Gégout et al. ${ }^{23}$ Obtaining nanocrystals that are compatible with organic solvents is vital for applications in optoelectronic devices. 
All these advantages have motivated research into watersoluble QDs in an attempt to increase the photoluminescence quantum yield $\left(\phi_{\mathrm{f}}\right)$ of materials synthesized in aqueous medium and to promote surface modifications that will ensure desirable optical and electronic properties as well as good chemical stability.

Regarding the synthesis of QDs via the water-based method, it is possible to alter the size-dependent features and structure of the nanocrystals by modifying a set of variables such as precursors concentration, reaction time, reaction medium $\mathrm{pH}$, reaction temperature, surface ligand type, and reactants molar ratio, among other parameters. ${ }^{6,8,17,18}$

Many methods such as high-resolution transmission electron microscopy (TEM), ${ }^{24}$ small-angle X-ray scattering, ${ }^{25}$ Raman scattering, ${ }^{25}$ and UV-vis absorption spectroscopy ${ }^{26}$ can help to determine the accurate size distribution of QDs. Unfortunately, these methods still have limitations like time-consuming sample preparation, limited instrument availability, laborious data processing, and low resolution. ${ }^{27}$ The color of the QD emission depends on nanocrystal size, while the width of the PL peak depends on nanocrystal size distribution. Hence, on the basis of previous studies, correlation fluorescence spectra could afford the maximum diameter of QDs. ${ }^{28}$

Peng et al. ${ }^{26}$ correlated the optical properties of QDs with nanocrystal size. In the case of high-quality $\mathrm{CdTe}, \mathrm{CdSe}$, and CdS nanocrystals, these authors found that the extinction coefficient per mole of nanocrystals at the first excitonic absorption peak heavily depended on crystal size (between a square and a cubic dependence). By compiling TEM data and the maximum of the first excitonic absorption peak, these authors established a useful empiric dependence that aided determination of the size and extinction coefficients of QDs. Mulvaney et al. ${ }^{29}$ re-examined the size-dependent optical absorption coefficients of CdSe nanocrystals at the band-edge and constructed calibration curves for the sizedependent first absorption peak and the molar extinction coefficient. These calibration curves helped to determine the concentration of nanocrystals in solution accurately.

Radotic et al. ${ }^{27}$ developed the first method to estimate the size of QDs on the basis of their emission properties. These authors employed statistical methods (EFA-evolving factor analysis and MCR-ALS-multivariate curve resolutionalternating least square) to decompose the emission spectra of nanoparticles and identify fractions of QDs with different diameters. Recent works have highlighted this methodology. ${ }^{28,30}$

Together, MCR-ALS ${ }^{31-33}$ and EFA ${ }^{34,35}$ are some of the most popular methods to deconvolution overlapping signals.

MCR-ALS can handle multiple arrays simultaneously, ${ }^{36}$ thereby reducing the rotational ambiguity intrinsic to bilinear methods and eliminating problems of rank deficiency. Moreover, MCR-ALS presents diverse and flexible constraints that help to improve resolution ${ }^{33}$. Finally, MCR-ALS has a very friendly graphical user interface ${ }^{37}$ with easy and free access through the MATLAB platform.
EFA is a time dependent rank analysis where the evolution of the total rank of a matrix $\mathrm{X}$ is followed by plotting the singular values of an increasing number of consecutive spectra as a function time ${ }^{34,35}$. This approach allows determination of the number of components, which here is related to the number of fraction of QDs with different distribution sizes, and gives an initial estimate of concentration profile.

In this perspective, this study deals with the synthesis, optical characterization and size distribution determined indirectly by curve resolution methods EFA and MCR-ALS applied to photoluminescence data of CdSe QDs in colloidal aqueous medium using different reaction times and different surface ligands.

\section{Experimental}

\subsection{Materials}

Selenium powder (100 mesh, 99.8\%), thioglycolic acid (TGA, 99\%), 3-mercaptopropionic acid (MPA, 99\%), glutathione (GSH, 99\%) and thioglycerol (TGH, 98\%) were obtained from Sigma-Aldrich (St. Louis, MO, USA). Sodium borohydride $\left(\mathrm{NaBH}_{4}, 97 \%\right)$ was acquired from Nuclear (São Paulo, Brazil). Cadmium chloride monohydrate $\left(\mathrm{CdCl}_{2} \cdot \mathrm{H}_{2} \mathrm{O}\right.$, 99\%) was provided by Vetec (Rio de Janeiro, Brazil). All the chemicals were used without additional purification. Milli-Q ultrapure water (Millipore Co., Billerica, MA, USA) was employed in the synthesis of nanoparticles.

\subsection{Synthesis of CdSe QDs}

The experimental procedure was performed on the basis of previous studies..$^{39,41,42,43}$ First, the selenium precursor was prepared in a three neck round-bottom flask by dissolving $0.4 \mathrm{mmol}$ of selenium and $1 \mathrm{mmol}$ of sodium borohydride in $10 \mathrm{~mL}$ of distilled water, under argon atmosphere and constant stirring. This solution was kept in an ice bath for $2 \mathrm{~h}$, until all the selenium was reduced. In a second reaction flask, $0.4 \mathrm{mmol}$ of cadmium chloride and $2 \mathrm{mmol}$ of ligand was added to $80 \mathrm{~mL}$ of distilled water; the $\mathrm{pH}$ was adjusted to 10.0 by addition of sodium hydroxide. Next, the selenium precursor solution, as prepared, was added to the latter reaction flask, and the resulting solution was stirred and heated $\left(100^{\circ} \mathrm{C}\right)$. Particle size was monitored throughout the process. To follow the progress of the synthesis, aliquots were removed from the reaction medium at regular time intervals and submitted to UV-Vis and PL analyses. Four different types of surface ligands were used: 3-mercaptopropionic acid (MPA), thioglycolic acid (TGA), thioglycerol (TGH) and glutathione (GSH) (Figure 1).

At the end of the heating and stirring process, the $\mathrm{CdSe}$ solution was separated from the reaction mixture. First, the as-prepared nanocrystal solution was concentrated to one fifth of its volume in a rotary evaporator attached 
<smiles>O=C(O)CCS</smiles><smiles>OCC(O)CS</smiles>

(TGH)

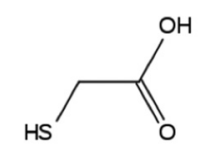

(TGA)

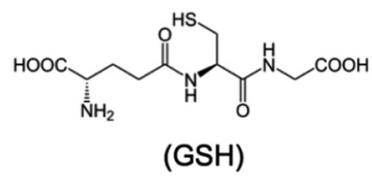

Figure 1: Structure of the capping agents: 3-mercaptopropionic (MPA), thioglycolic acid (TGA), thioglycerol (TGH), and glutathione (GSH).

to a vacuum pump. Then, a small portion of acetone was added to the solution, to remove impurities and other reaction products. The supernatant of this solution was separated by centrifugation, and acetone was added to the supernatant again, until the solution became cloudy. Further centrifugation allowed larger CdSe particles to precipitate. The precipitated particles were dried at $40^{\circ} \mathrm{C}$, in an oven.

\subsection{Characterization}

UV-Vis absorption and fluorescence at single excitation wavelength spectra were acquired on a Varian Cary 50 spectrophotometer and on a Shimadzu RF-5301 PC spectrofluorophotometer, respectively. Absorption and fluorescence measurements were performed in quartz cuvettes with an optical path length of $10 \mathrm{~mm}$ (Shimadzu). Spectra of air-saturated solutions were recorded at room temperature. X-ray diffraction (XRD) patterns of the CdSe QD samples sprayed in acetone were registered in a nonreflective silicon sample port with the aid of a Shimadzu XRD-6000 diffractometer operating in the scanning mode $\left(15^{\circ}-60^{\circ}\right)$ and using $\mathrm{CuK} \alpha$ radiation $(\lambda=1.5418 \AA)$ generated at $40 \mathrm{kV}$ and at a current of $30 \mathrm{~mA}$. Steps of $0.02^{\circ}$ and a sampling time of $4 \mathrm{~h}$ were employed. Divergence slits of $1.0 \mathrm{~mm}$, scattering slits of $1.0 \mathrm{~mm}$, and receiving slits of $0.3 \mathrm{~mm}$ were used. Fourier-transform infrared spectroscopy (FT-IR) was conducted on a spectrometer Spectrum GX Perkin Elmer. The spectra were obtained in the transmission mode in the range of 400 to $4000 \mathrm{~cm}^{-1}$ with accumulation of 32 spectra. The resolution was $2 \mathrm{~cm}^{-1}$. Additionally, the multi-wavelength fluorescence data were obtained from the successive acquisition of emission spectra at multiple excitation wavelengths, generating excitation-emission matrices (EEM) for CdSe QDs bearing distinct surface ligands. For CdSe/MPA QDs, the EEM were collected for each time of synthesis with spectral ranges of 440-540 nm for excitation and 550-900 $\mathrm{nm}$ for emission. For CdSe/ $\mathrm{GSH}$, the spectral range were $410-510 \mathrm{~nm}$ for excitation and $400-900 \mathrm{~nm}$ for emission.

\subsection{Size distribution determination of CdSe QDs}

The prepared CdSe QDs were re-dispersed in water prior to the absorption and fluorescence emission experiments. First, the CdSe QD sizes were determined by UV-Vis spectroscopy with the aid of the equations obtained by Peng et al. ${ }^{26}$ To this end, samples were transferred to quartz cuvettes, and the absorption spectrum was recorded. Calibration curves were constructed to correlate the size of CdSe QDs with the maximum fluorescence.

The successive acquisition of emission spectra at multiple excitation wavelengths, which generates excitation-emission matrices (EEM), provides full spectral information and is more accurate than absorption spectra. In this sense, specific measurement procedures were applied to detect any signal of fluorescence for $\mathrm{CdSe}$ QDs bearing distinct surface ligands.

On average, ${ }^{21-35}$ emission spectra were collected for each CdSe QDs dispersion obtained at different synthesis time of reaction by varying the excitation wavelength by $5 \mathrm{~nm}$. To include all the geometric characteristics of the emission spectrum, for each sample, the initial excitation wavelength was defined in such a way that recording of the emission spectra began between 50 and $60 \mathrm{~nm}$ before the emission increased sharply.

In order, to get a complete spectral information of each CdSe QDs dispersion, excitation-emission matrices were also collected at different times of the synthesis $-15,30$, $60,90,120,150$, and $180 \mathrm{~min}$. For the matrices whose excitation and emission wavelengths were overlapped, it was necessary to accomplish a pre-treatment that eliminated the elastic Rayleigh scattering. This pre-treatment excluded the affected region and recreated this area by interpolation. ${ }^{44}$ Then, the matrices were arranged into single ones by matrix column-augmentation (X = [X15; X30; X60; X90; X120; $\mathrm{X} 150 ; \mathrm{X} 180])$, and curve resolution methods were applied to investigate the CdSe QDs size distribution and determine the number of fraction with different particle size.

MCR-ALS is an iterative curve resolution method that focuses on the bilinear decomposition of mixed multicomponent information store in a data matrix into chemically meaningful pure contributions of each of the components in the mixture. ${ }^{31-33}$ The bilinear decomposition of the experimental data matrix is performed using the model equation:

$$
\mathrm{X}=\mathrm{CS}^{\mathrm{T}}+\mathrm{E}
$$

where $\mathrm{C}$ is the matrix that describes how the excitation spectra of the $n$ QDs changes along the time, and $\mathrm{S}^{\mathrm{T}}$ is the matrix that represents the pure emission spectra of the $n$ QDs of the data matrix. E is the residual matrix corresponding to the variance not explained by the model $\mathrm{CS}^{\mathrm{T}}$.

The first step in the resolution procedure was to determine the number of components, $n$, causing the observed variance in X. The second step was to obtain an initial estimate 
of the concentration matrix. In this work, the number of components, $n$, and, the initial estimate of concentration matrix were obtained by the EFA method. ${ }^{40-41}$ The third step referred to the MCR-ALS decomposition step. At this stage, the restriction of non-negativity of the spectral profiles was implemented. The MCR-ALS program ${ }^{37}$ implemented in the MATLAB 7.0 platform was used for all calculations.

\section{Results and Discussion}

\subsection{CdSe $Q D s$}

A colloidal approach that used distinct surface ligands afforded different CdSe QDs in aqueous medium. A comparative study of these CdSe QDs showed how the surface ligands TGA, MPA, GSH, and TGH affected the optical properties of the nanocrystals.

To obtain the CdSe QDs, the initial step was to react metallic selenium and sodium borohydride, to achieve the NaHSe precursor as represented by reaction (1):

$$
\begin{aligned}
& 4 \mathrm{NaBH}_{4}+2 \mathrm{Se}+7 \mathrm{H}_{2} \mathrm{O} \rightarrow \\
& 2 \mathrm{NaHSe}+\mathrm{Na}_{2} \mathrm{~B}_{4} \mathrm{O}_{7}+14 \mathrm{H}_{2}
\end{aligned}
$$

Then, the NaHSe precursor was added to a solution containing the surface ligand and $\mathrm{Cd}^{2+}$ ions, to give the $\mathrm{CdSe}$ nanoparticles according to reaction (2):

$$
\begin{aligned}
& \mathrm{NaHSe}+\mathrm{CdCl}_{2} \rightarrow \\
& \mathrm{CdSe}+\mathrm{Na}^{+}+2 \mathrm{Cl}^{-}+\mathrm{H}^{+}
\end{aligned}
$$

Figure 2 depicts the UV-vis and fluorescence spectra that helped to monitor the progress of the synthesis of CdSe QDs bearing MPA as surface ligand (UV-vis and fluorescence spectra of CdSe QDs synthesized using GSH, TGA e TGH as surface ligands are shown in Figures 3, 4 and 5). For all the samples, the excitonic absorption and luminescence bands shifted slightly toward lower energies with increasing particle size. This shift was consistent with quantum confinement effects and agreed with literature results published for similar systems. ${ }^{45}$ Figure 2 a revealed slightly red-shifted absorption bands on going from the aliquot withdrawn at $15 \mathrm{~min}$ of synthesis, $482 \mathrm{~nm}$, to the aliquot withdrawn at $180 \mathrm{~min}$ of synthesis, $493 \mathrm{~nm}$. This shift suggested that nanoparticle size increased along the synthesis. Similarly, the PL spectra, obtained at excitation wavelength of $355 \mathrm{~nm}$, in Figure $2 \mathrm{~b}$ showed slight displacement of the emission bands toward longer wavelengths, from $649 \mathrm{~nm}$ in the case of the first aliquot to $659 \mathrm{~nm}$ at the end of the synthesis. This displacement corresponded to the emission of orange color.

Figure 6 illustrates the temporal evolution of the position of the absorption and emission bands for the different surface ligands used during the growth of CdSe QDs. GSH shifted the absorption $(\sim 53 \mathrm{~nm})$ and emission $(\sim 140 \mathrm{~nm})$ bands toward the red the most. In other words, this capping agent provided higher nanoparticle growth rate as compared with the other capping agents. This faster growth may have originated from the higher steric hindrance and the larger susceptibility to thermal decomposition of GSH as compared with the other ligands. ${ }^{39}$ Hence, GSH was more turned off from the surface of the QDs ("off” state). Conversely, TGH displaced the absorption $(\sim 10 \mathrm{~nm})$ and emission $(\sim 6 \mathrm{~nm})$ bands the least, which indicated that this ligand promoted the slowest kinetic growth of CdSe nanocrystals. Probably, the two close functional $\mathrm{OH}$ groups in the structure of TGH caused this ligand to be more strongly attached to the surface of the nanocrystals ("on" state), thereby preventing a large increase in particle size. As for MPA and TGA, results were similar and lay between the behaviors of GSH and TGH, as seen from the shifts in the absorption $(\sim 20 \mathrm{~nm}$ and $\sim 29$ $\mathrm{nm}$ for MPA and TGA, respectively) and emission ( $\sim 12 \mathrm{~nm}$ and $\sim 13 \mathrm{~nm}$ for MPA and TGA, respectively) bands. For TGA-capped QDs a NIR (near infra-red) emission could be related to the surface-defects that generate shallow levels near the valence band, that acts as traps for photogenerated holes. ${ }^{46,47}$ The obtained results agreed with literature data presented for CdTe QDs obtained in aqueous medium and bearing GSH, MPA, TGA, and TGH as surface ligands. ${ }^{39}$

Figure 7 brings the XRD patterns registered for the $\mathrm{CdSe}$ QDs stabilized with the studied ligands. The diffraction patterns displayed the three main diffraction peaks of CdSe, at $2 \theta=25.1,41.8$, and 49.2 degrees, which confirmed the crystallinity of the CdSe QDs. The positions of the diffraction peaks standing for the (111), (220), and (311) planes referred to the zinc blend structure and matched the standard pattern well (JCPDS No.- 00-019-0191). ${ }^{48,49}$ Among the employed surface ligands, GSH shifted the diffraction peaks toward the CdS cubic crystalline phase the most. This happened because GSH was more susceptible to thermal decomposition and probably passivated the QDs surface more effectively. This may have increased sulfur release on the surface of the CdSe nanocrystals and may even have generated a thin CdS layer, to give a quasi core-shell structure. ${ }^{39,49}$ According to the literature, CdTe QDs capped with GSH in aqueous medium display the same behavior. ${ }^{39}$

Infrared spectroscopy furnished information about the bond that originated between the surface ligands and the $\mathrm{CdSe}$ surface. For illustration, Figure 8 contains the infrared spectra of the pure MPA, TGA, TGH, and GSH ligands and of the CdSe QDs stabilized with these ligands. Additionally, Figure 8 presents the assignment of the main FT-IR band and provides a scheme that represents how the surface ligands bind to the CdSe QDs.

The surface ligands alone had an absorption band around $2500 \mathrm{~cm}^{-1}$, due to the $\mathrm{S}-\mathrm{H}$ group. This band disappeared in the case of the capped QDs, indicating that the surface ligands attached to the surface of CdSe QDs via their sulfur atoms. The pure ligands also presented a strong band at $\sim 1710 \mathrm{~cm}^{-1}$, 

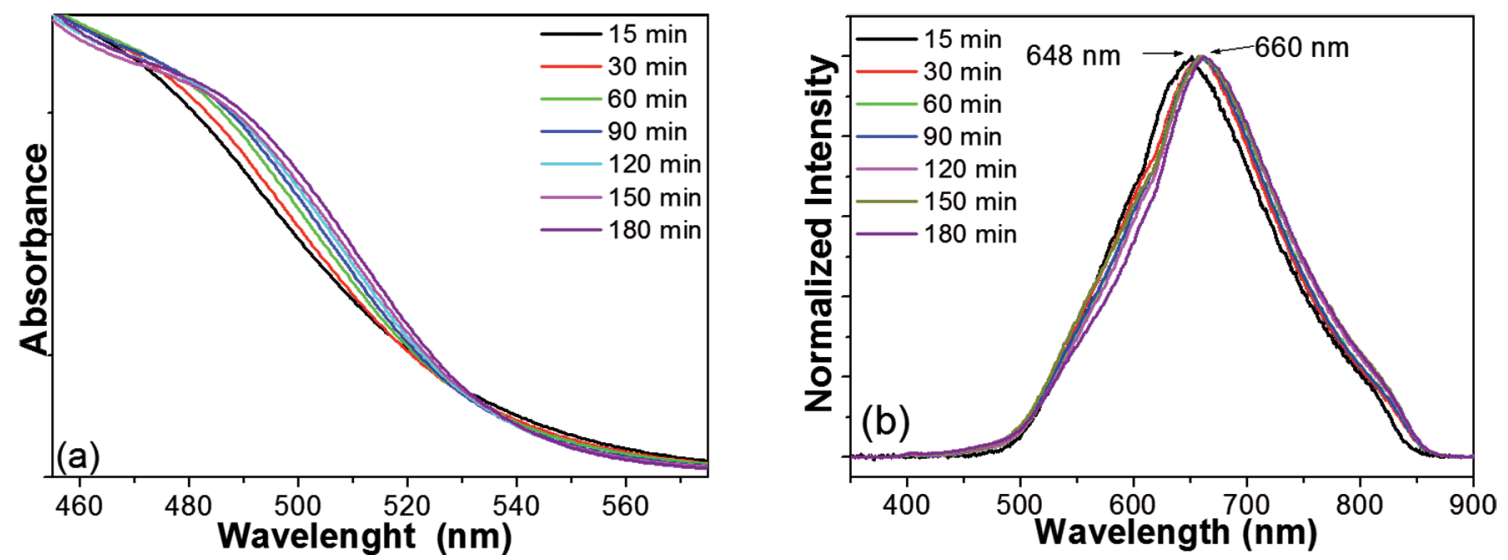

Figure 2: UV-visible absorption (a) and fluorescence (b) spectra of CdSe/MPA QDs at different reaction times.
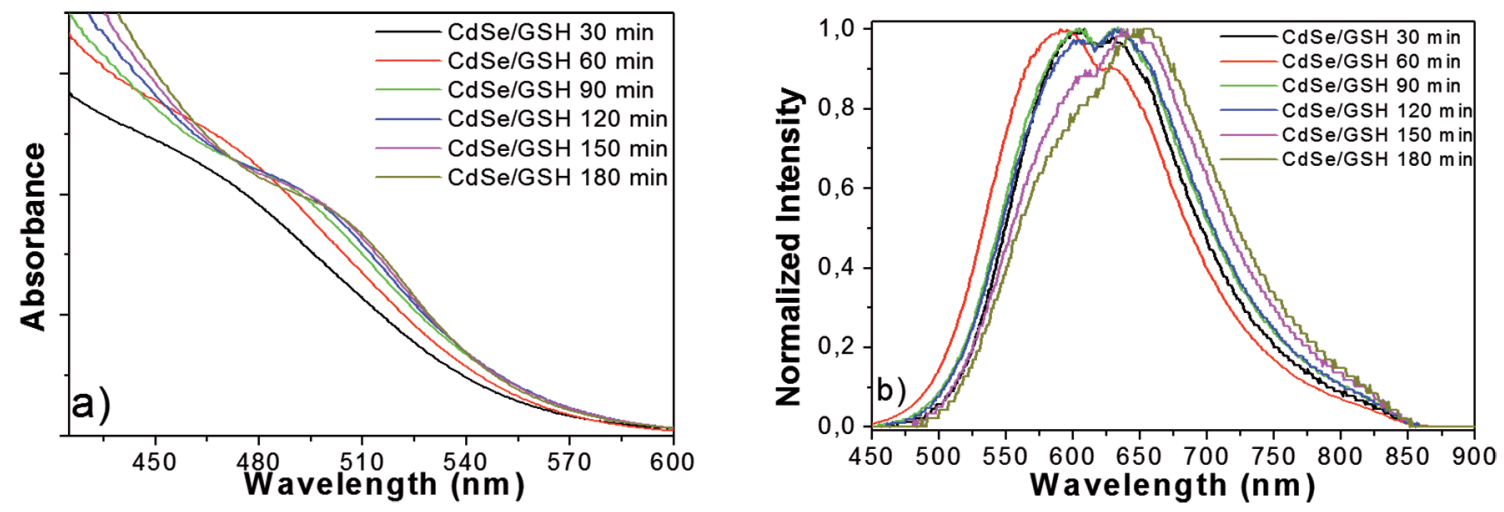

Figure 3: UV-visible absorption a) and fluorescence b) spectra of CdSe/GSH QDs at different reaction times.
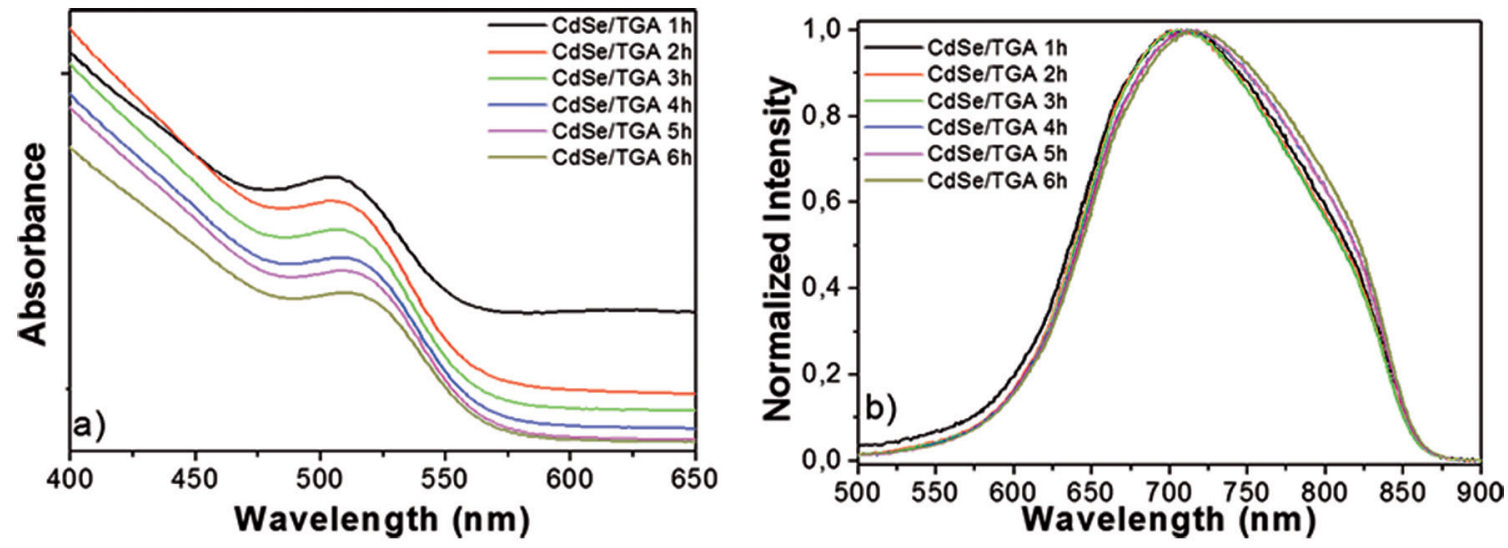

Figure 4: UV-visible absorption a) and fluorescence b) spectra of CdSe/TGA QDs at different reaction times.

due to $\mathrm{C}-\mathrm{O}$ stretching. In contrast, the spectra of the capped CdSe samples displayed the symmetric and asymmetric absorption bands assigned to the carboxylate anion of the surface ligands. The existence of the carboxylate anion in the QDs resulted from the synthesis process, which used $\mathrm{NaOH}$ to adjust the $\mathrm{pH}$ to 10.0 and therefore deprotonated the $\mathrm{COOH}$ group.
The $\mathrm{O}-\mathrm{H}$ stretching of absorbed water emerged as a broad band in the region of $3300-2900 \mathrm{~cm}^{-1}$ in the spectra of MPA-capped CdSe QDs, TGA-capped CdSe QDs, and TGH-capped CdSe QDs, whereas the spectrum of GSHcapped CdSe QDs exhibited a broad band in the region of $3500-3100 \mathrm{~cm}^{-1}$, attributed to N-H stretching. The spectrum of TGH-capped CdSe QDs also had absorption 

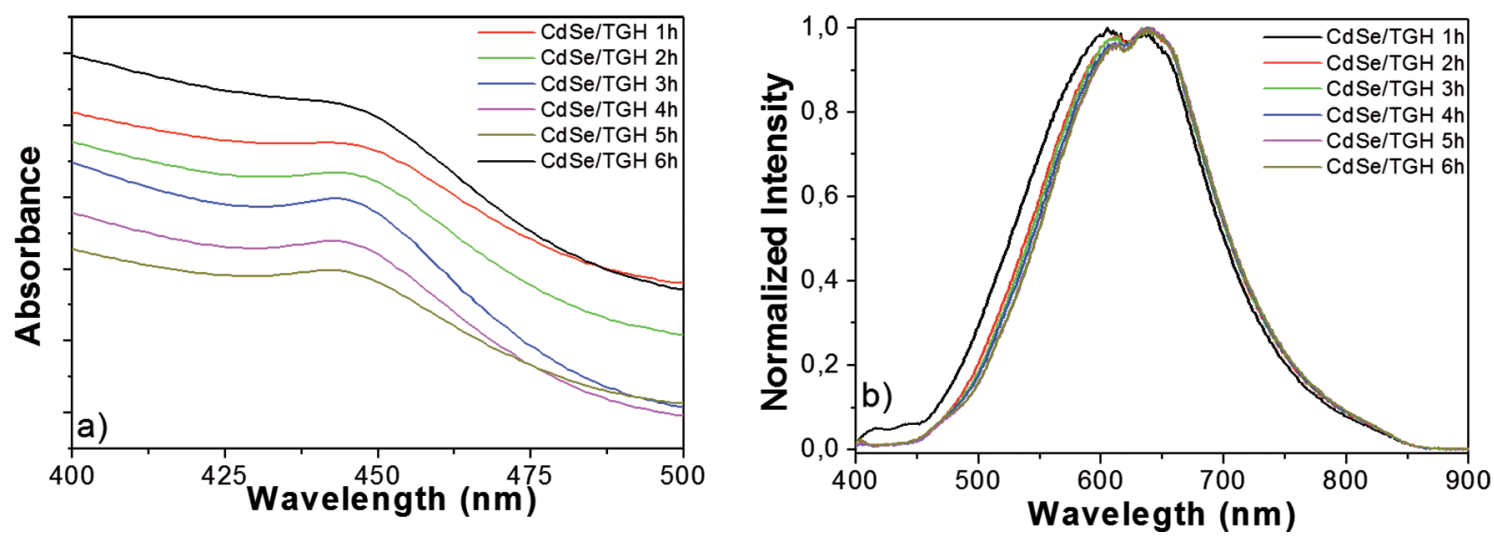

Figure 5: UV-visible absorption a) and fluorescence b) spectra of CdSe/TGH QDs at different reaction times.
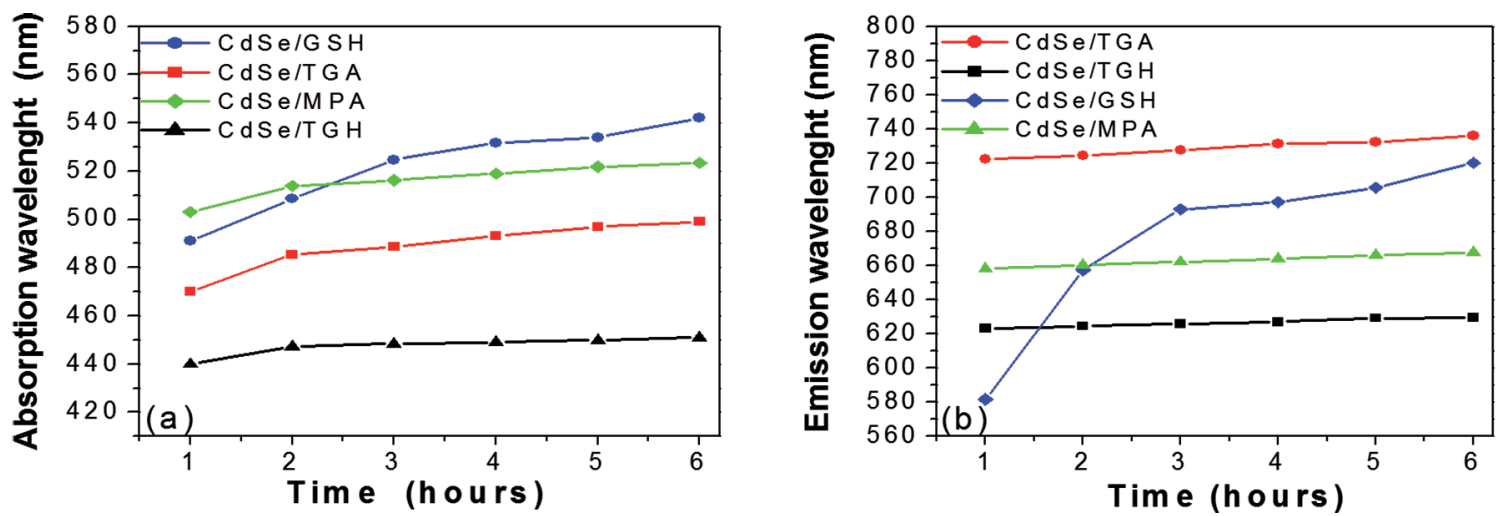

Figure 6: Temporal evolution of the maximum (a) absorption and (b) emission peaks of CdSe QDs with MPA, GSH, TGA, and TGH surface ligands.

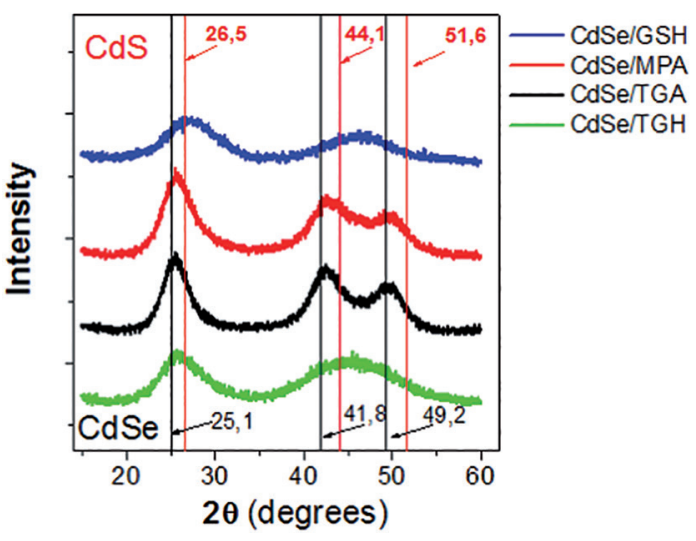

Figure 7: XRD patterns of CdSe QDs capped with MPA, GSH, TGA, and TGH. The graphic shows the diffraction peak positions for CdSe (bottom pattern) and CdS (upper pattern).

bands around 1500 and $900 \mathrm{~cm}^{-1}$, ascribed to $\mathrm{O}-\mathrm{H}$ bending and $\mathrm{C}-\mathrm{O}$ stretching. ${ }^{50,51}$ The FT-IR assignments agreed with data recorded for similar systems such as watersoluble CdTe, which means that CdSe and CdTe QDs interact with thiol surface ligands in aqueous medium in much the same way.

\subsection{Size determination of CdSe QDs}

The absorption wavelength $\left(\lambda_{a b s}\right)$ obtained by UV-Vis spectroscopy and the equation obtained by Peng et al. ${ }^{26}$ aided size determination of CdSe QDs. Table 1 shows the correlation between the calculated diameters and the maximum emission wavelength for MPA-capped CdSe QDs, at excitation wavelength of $355 \mathrm{~nm}$.

The data presented in Table I allowed construction of a calibration curve that related the position of the maximum fluorescence with the diameter calculated for the nanoparticle (see Figure 9 for the calibration curves constructed for MPA-capped CdSe QDs and GSH-capped CdSe QDs). A polynomial fit of the experimental data provided a better calibration curve, yielded the highest coefficient of determination ${ }^{28}\left(\mathrm{R}^{2}\right)$ and the deviation from the data, and gave a fine description of the system in the treated interval. In this way, by the calibration curve equation, and knowing the sample maximum fluorescence position, is possible to estimate the average particles sizes.

Fluorescence was measured for each capped CdSe QDs sample at different times; i.e., at 15, 30, 60, 90, 120, 150, and 180 min of synthesis. Because QDs of different sizes should 

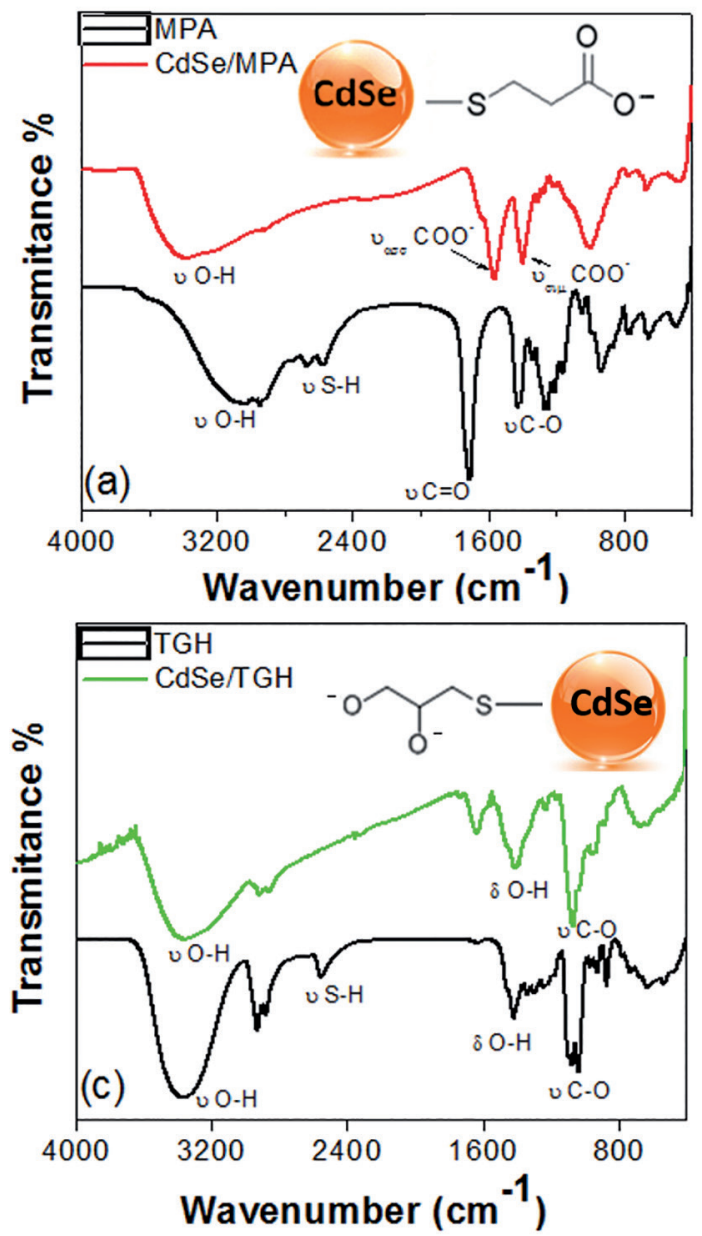
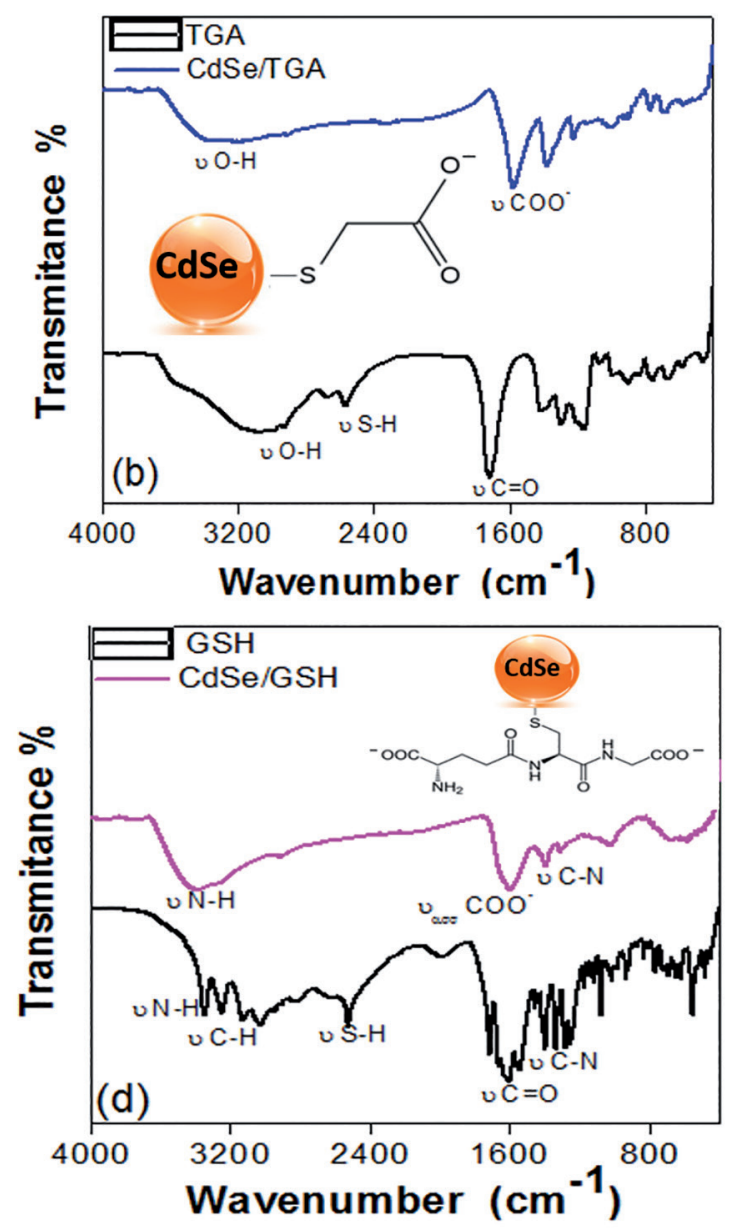

Figure 8: Infrared spectra of CdSe QDs capped with (a) MPA, (b) TGA, (c) TGH, and (d) GSH.

Table 1: Position of the maximum fluorescence in relation to the diameter of MPA-capped CdSe QDs.

\begin{tabular}{lcc}
\hline & $\begin{array}{c}\text { Maximum fluorescence } \\
\text { position }(\mathrm{nm})\end{array}$ & Particle diameter $(\mathrm{nm})$ \\
\hline $15 \mathrm{~min}$ & 648 & 2.07 \\
$30 \mathrm{~min}$ & 652 & 2.16 \\
$60 \mathrm{~min}$ & 653 & 2.18 \\
$90 \mathrm{~min}$ & 654 & 2.19 \\
$120 \mathrm{~min}$ & 655 & 2.21 \\
$150 \mathrm{~min}$ & 658 & 2.22 \\
$180 \mathrm{~min}$ & 660 & 2.23 \\
\hline
\end{tabular}

emit in different positions of the spectrum, this procedure helped to determine the size distributions of the QDs along the synthesis. In addition, the samples were irradiated with different wavelengths by varying the excitation wavelength by $5 \mathrm{~nm}$ each time the spectrum was recorded. Figure 10 illustrates the results for $30 \mathrm{~min}$ MPA-capped CdSe QDs and 30 min GSH-capped CdSe QDs.

Decomposition by the MCR-ALS method of the set of spectra obtained for MPA-capped CdSe QDs revealed the existence of two types of QDs with specific diameters in this material (Figure 11a). More specifically, MPA-capped CdSe QDs displayed maximum emission at 642 and 686 $\mathrm{nm}$, which corresponded to calculated diameters of $2.74 \pm$ 0.1 and $3.05 \pm 0.1 \mathrm{~nm}$, the calculated average particles sizes, obtained after the distribution of sizes calculations, presents an error of $0.1 \mathrm{~nm}$, due to the calibration curve equation error, as inferred from the plot depicted in Figure 9. As for GSH-capped CdSe QDs, maximum emission emerged at 582 and $658 \mathrm{~nm}$, which corresponded to diameters of 1.94 \pm 0.1 and $2.32 \pm 0.1 \mathrm{~nm}$, respectively (Figure $11 \mathrm{~b}$ ). With respect to the synthesis method, and synthesis time, GSHcapped CdSe QDs and MPA-capped CdSe QDs showed the same size distribution with two resolved emission spectra for each system. This is an important result: while the very large bandwidths values of water-soluble CdSe are mainly associated with broad size distribution, here there were only two main sizes of capped CdSe QDs at different times of synthesis. Therefore, the structure and shape of the nanocrystals in the CdSe QDs samples synthesized in this work were similar. Actually, the different thiol-based surface ligands affected the nanocrystal growth rate, but they did not influence the nanocrystal size distribution. 

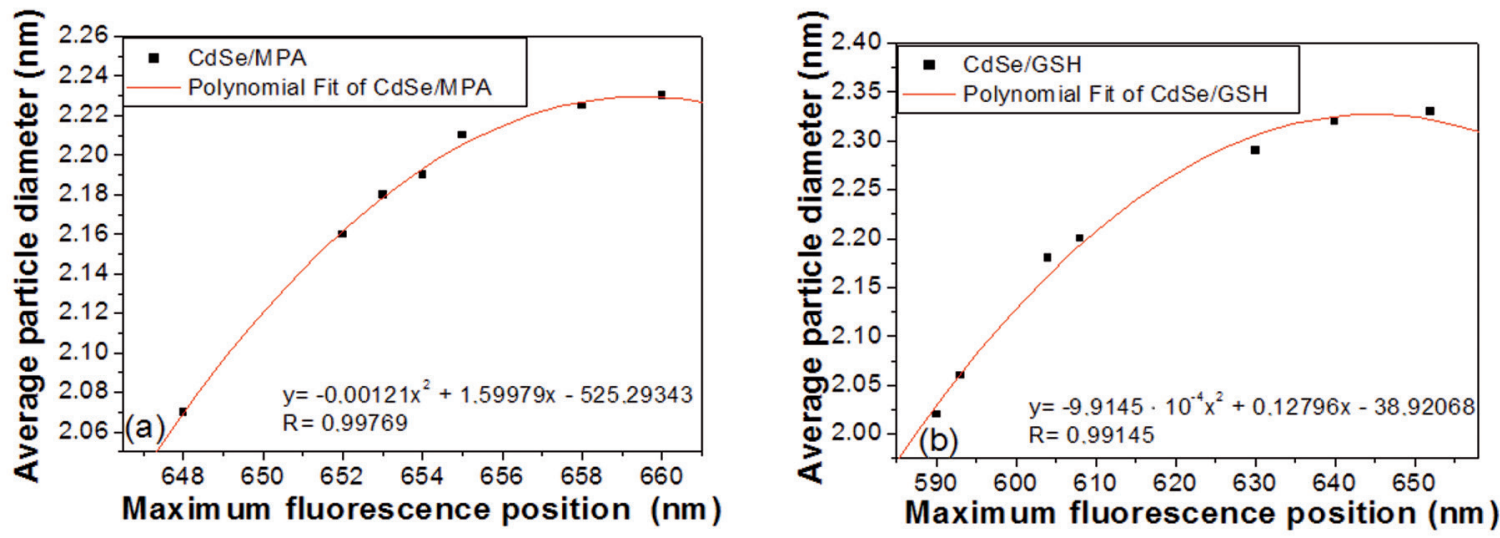

Figure 9: Calibration curves for the position of maximum fluorescence versus the diameter calculated for QDs: (a) MPA-capped CdSe QDs and (b) GSH-capped CdSe QDs.
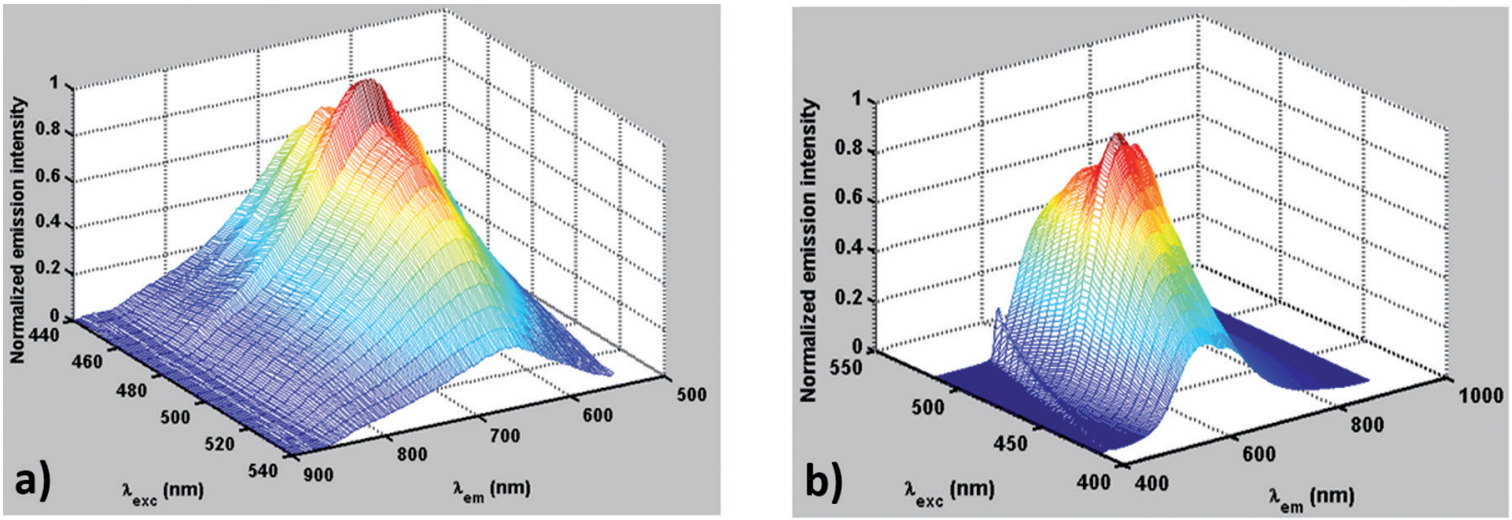

Figure 10: Emission spectra of (a) MPA-capped CdSe QDs and (b) GSH-capped CdSe QDs obtained at 30 min of synthesis, after excitation at various wavelengths.
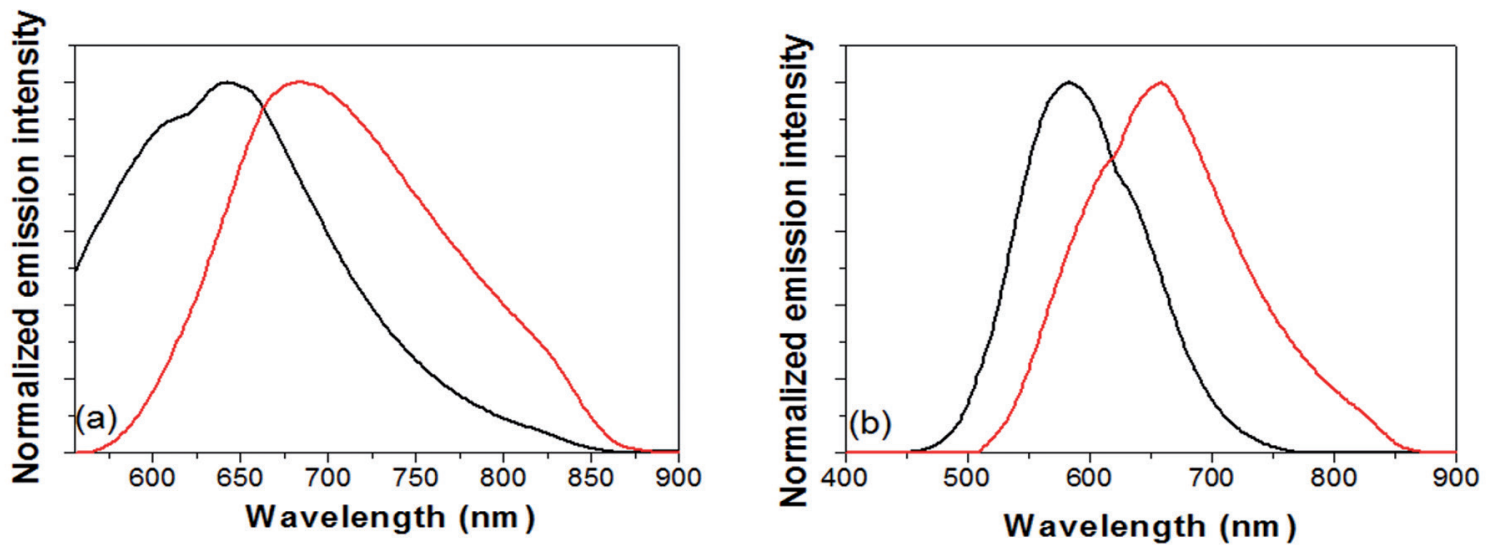

Figure 11: Component spectra for CdSe QDs bearing different surface ligands: (a) MPA and (b) GSH.

\section{Conclusions}

This work described the successful synthesis of capped CdSe QDs via a chemical colloidal process conducted in aqueous medium, in the presence of one of the following surface ligands: thioglycolic acid (TGA), 3-mercaptopropionic acid (MPA), glutathione (GSH), or thioglycerol (TGH). A comparative study showed that the MPA- and TGA-capped
CdSe QDs displayed similar properties. As for the TGH ligand, it bears two functional $\mathrm{OH}$ groups in close proximity in its structure. These groups promoted strong interactions between TGH and the nanocrystals surface, preventing CdSe growth. The size of the GSH ligand provided faster CdSe QDs growth. In addition, the thermal instability of GSH during the synthesis of capped CdSe QDs released sulfur on the 
surface of the nanocrystals and generated a quasi $\mathrm{CdSe} / \mathrm{CdS}$ core/shell structure. The capping agent probably attached to the CdSe QDs surface via the - SH end of the molecule.

Decomposition of the spectra of the various CdSe QDs by chemometric techniques afforded the size distribution of the nanoparticles obtained during the synthesis. GSH-capped CdSe QDs and MPA-capped CdSe QDs showed the same size distribution, with two resolved emission spectra for each surface ligand. The maximum emission bands obtained for GSH-capped CdSe QDs were 582 and $658 \mathrm{~nm}$, which corresponded to QDs with diameters of 1.94 and $2.32 \mathrm{~nm}$, respectively. As for MPA-capped CdSe QDs, the maximum emission bands were 642 and $686 \mathrm{~nm}$, referring to QDs with diameters of 2.74 and $3.05 \mathrm{~nm}$, respectively.

\section{Acknowledgements}

The authors acknowledge to Coordenação de Aperfeiçoamento de Pessoal de Nível Superior (CAPES), Fundação de Amparo à Pesquisa do Estado de Minas Gerais (FAPEMIG), and Conselho Nacional de Desenvolvimento Científico e Tecnológico (CNPq). This work is a collaboration research project of members of the Rede Mineira de Química (RQ-MG) supported by FAPEMIG (Project: CEX - RED00010-14).

\section{References}

1. Wang L, Cao L, Su G, Liu W, Xia C, Zhou H. Preparation and characterization of water-soluble $\mathrm{ZnSe}: \mathrm{Cu} / \mathrm{ZnS}$ core/shell quantum dots. Applied Surface Science. 2013;208:673-678.

2. Hetsch F, Xu X, Wang H, Kershaw SV, Rogach AL. Semiconductor Nanocrystal Quantum Dots as Solar Cell Components and Photosensitizers: Material, Charge Transfer, and Separation Aspects of Some Device Topologies. The Journal of Physical Chemistry Letters. 2011;2(15):1879-1887.

3. Kamat PV. Quantum Dot Solar Cells. The Next Big Thing in Photovoltaics. The Journal of Physical Chemistry Letters. 2013;4(6):908-918.

4. Rühle S, Shalom M, Zaban A. Quantum-dot-sensitized solar cells. ChemPhysChem. 2010;11(11):2290-2304.

5. Hines DA, Kamat PV. Quantum Dot Surface Chemistry: Ligand Effects and Electron Transfer Reactions. The Journal of Physical Chemistry C. 2013;117(27):14418-14426.

6. Alivisatos AP, Gu W, Larabell C. Quantum dots as cellular probes. Annual Review of Biomedical Engineering. 2005;7:55-76.

7. Hao E, Zhang H, Yang B, Ren H, Shen J. Preparation of luminescent polyelectrolyte/Cu-doped $\mathrm{ZnSe}$ nanoparticle multilayer composite films. Journal of Colloid and Interface Science. 2001;238(2):285-290.

8. Manzoor K, Jonhy S, Thomas D, Setua S, Menon D, Nair S. Bio-conjugated luminescent quantum dots of doped ZnS: a cytofriendly system for targeted cancer imaging. Nanotechnology. 2009;20:065102.
9. Silva FO, Viol LCS, Ferreira DL, Alves JLA, Schiavon MA. O estado da arte da síntese de semicondutores nanocristalinos coloidais. Química Nova. 2010;33(9):1933-1939.

10. Robel I, Subramanian V, Kuno M, Kamat PV. Quantum Dot Solar Cells. Harvesting Light Energy with CdSe Nanocrystals Molecularly Linked to Mesoscopic TiO2 Films. Journal of the American Chemical Society. 2006;128(7):2385-2393.

11. Mattoussi H, Radzilowski LH, Dabbousi BO, Thomas EL, Bawendi MG, Rubner MF. Electroluminescence from heterostructures of poly(phenylene vinylene) and inorganic CdSe nanocrystals. Journal of Applied Physics. 1998;83(12):7965.

12. Kagan CR, Nirmal MM, Bawendi MG. Electronic Energy Transfer in CdSe Quantum Dot Solids. Physical Review Letters. 1996;76:1517.

13. Oertel DC, Bawendi MG, Arango AC, Bulović V. Photodetectors based on treated CdSe quantum-dot films. Applied Physics Letters. 2005;87(21):213505.

14. Patil P, Laltlanzuala C, Datta S. Sensitized solar cell from type-II CdTe/CdSe core/shell nanocrystals synthesized without seed purification at low temperature. Journal of Alloys and Compounds. 2014;607:230-237.

15. Ekimov AI, Onuschenko AA. Quantum size effect in the optical spectra of semiconductor microcrystals. Soviet Physics: Semiconductors. 1982;16(7):1215-1219.

16. Daudin B, Widmann F, Fenillet G, Samson Y, Rouvière JL, Pelekanos N. Self organization of nitride quantum dots by molecular beam epitaxy. Materials Science and Engineering: B. 1999;59(1-3):330-334.

17. Murray CB, Norris DJ, Bawendi MG. Synthesis and characterization of nearly monodisperse $\mathrm{CdE}$ ( $\mathrm{E}=$ sulfur, selenium, tellurium) semiconductor nanocrystallites. Journal of the American Chemical Society. 1993;115(19):8706-8715.

18. Reiss P, Bleuse J, Pron A. Highly Luminescent CdSe/ZnSe Core/Shell Nanocrystals of Low Size Dispersion. Nano Letters. 2002;2(7):781-784.

19. Mi W, Tian W, Tian J, Jia J, Liu X, Dai J, et al. Synthesis of CdSe quantum dots in ethanol: A facile way to achieve photoluminescence with high brightness. Colloids and Surfaces A: Physicochemical and Engineering Aspects. 2013;417:179-182.

20. Osbourn G. Strained-layer superlattices: A brief review. IEEE Journal of Quantum Electronics. 1986;22(9):1677-1681.

21. Sah CT. Fundamentals of Solid-State Electronics. Singapore: World Scientific; 1991.

22. Zhang H, Cui Z, Wang Y, Zhang K, Ji X, Lü C, et al. From water-soluble CdTe nanocrystal to fluorescent nanocrystalpolymer transparent composites using polymerizable surfactants. Advanced Materials. 2003;15(10):777-780.

23. Gégout C, McAtee ML, Bennett NM, Viranga Tillekeratne LM, Kirchhoff JR. Synthesis and characterization of luminescent cadmium selenide/zinc selenide/zinc sulfide cholinomimetic quantum dots. Nanoscale. 2012;4(15):4719-4725.

24. Chen S, Zhang X, Zhao Y, Zhang Q. Effects of reaction temperature on size and optical properties of CdSe nanocrystals. Bulletin of Materials Science. 2010;33(5):547-552. 
25. Dzhagan VM, Valakh MY, Raevshaya AE, Stroyuk AL, Kuchmiy SY, Zahn DRT. Resonant Raman scattering study of CdSe nanocrystals passivated with $\mathrm{CdS}$ and $\mathrm{ZnS}$. Nanotechnology. 2007;18(28):285701.

26. Yu WW, Qu LH, Guo WZ, Peng XG. Experimental Determination of the Extinction Coefficient of CdTe, CdSe, and CdS Nanocrystals. Chemistry of Materials. 2003;15(14):2854-2860.

27. Mutavdžić D, Xu J, Thakur G, Triulzi R, Kasas S, Jeremić M, et al. Determination of the size of quantum dots by fluorescence spectroscopy. Analyst. 2011;136(11):2391-2396.

28. Hormozi-Nezhad RM, Jalali-Heravi M, Kafrashi F. Detecting intermediate particles in the growth of coloidal zinc oxide nanoparticles in different chemical routes using MCR-ALS. Journal of Chemometrics. 2013;27(10):353-358.

29. Jasieniak J, Smith L, van Embden J, Mulvaney P, Califano M. Re-examination of the Size-Dependent Absorption Properties of CdSe Quantum Dots. The Journal of Physical Chemistry C. 2009;113(45):19468-19474.

30. Sousa JCL, Vivas MG, Ferrari JL, Mendonça CR, Schiavon MA. Determination of particle size distribution of water-soluble CdTe quantum dots by optical spectroscopy. RSC Advances. 2014;4(68):36024-36030.

31. Tauler R, Kowalski B, Heming S. Multivariate curve resolution applied to spectral data from multiple runs of an industrial process. Analytical Chemistry. 1993;65(15):2040-2047.

32. Tauler R. Calculation of maximum and minimum band boundaries of feasible solutions for species profiles obtained by multivariate curve resolution. Journal of Chemometrics. 2001;15(8):627-646.

33. de Juan A, Tauler R. Multivariate curve resolution (MCR) from 2000: progress in concepts and applications. Critical Reviews in Analytical Chemistry. 2006;36(3-4):163-176.

34. Maeder M, Zuberbuehler AD. The resolution of overlapping chromatographic peaks by evolving factor analysis. Analytica Chimica Acta. 1986;181:287-291.

35. Maeder M, Zilian A. Evolving factor analysis, a new multivariate technique in chromatography. Chemometrics and Intelligent Laboratory Systems. 1988;3(3):205-213.

36. Dantas C, Tauler R, Ferreira MMC. Exploring in vivo violacein biosynthesis by application of multivariate curve resolution on fused UV-vis absorption, fluorescence, and liquid chromatographymass spectrometry data. Analytical and Bioanalytical Chemistry. 2013;405(4):1293-1302.

37. Jaumont J, Gargallo R, de Juan A, Tauler R. A graphical userfriendly interface for MCR-ALS: a new tool for multivariate curve resolution in Matlab. Chemometrics and Intelligent Laboratory Systems. 2005;76(1):101-110.

38. van Embden J, Jasieniak J, Gómez DE, Mulvaney P, Giersig M. Review of the Synthetic Chemistry Involved in the Production of Core/Shell Semiconductor Nanocrystals. Australian Journal of Chemistry. 2007;60(7):457-471.
39. Silva FO, Carvalho MS, Mendonça R, Macedo WA, Balzuweit $\mathrm{K}$, Reis Pet al. Effect of surface ligands on the optical properties of aqueous soluble CdTe quantum dots. Nanoscale Research Letters. 2012;7:536.

40. Willians ATR, Winfield SA, Miller JN. Relative fluorescence quantum yields using a computer-controlled luminescence spectrometer. Analyst. 1983;108(1290):1067-1071.

41. Zhang H, Zhou Z, Yang B, Gao M. The Influence of Carboxyl Groups on the Photoluminescence of Mercaptocarboxylic Acid Stabilized CdTe Nanoparticles. The Journal of Physical Chemistry B. 2003;107(1):8-13.

42. Oluwafemi SO, Revaprasadu N, Ramirez AJ. A novel one-pot route for the synthesis of water-soluble cadmium selenide nanoparticles. Journal of Crystal Growth. 2008;310(13):3230-3234.

43. Aldeek F, Mustin C, Balan L, Medjahdi G, Roques-Carmes T, Arnoux P, et al. Enhanced Photostability from $\mathrm{CdSe}(\mathrm{S}) /$ $\mathrm{ZnO}$ Core/Shell Quantum Dots and Their Use in Biolabeling. European Journal of Inorganic Chemistry. 2011;2011(6):794-801.

44. Bahram M, Bro R, Stedmon C, Afkhami A. Handling of Rayleigh and Raman scatter for PARAFAC modeling of fluorescence data using interpolation. Journal of Chemometrics. 2006;20(34):99-105.

45. Amelia M, Impellizzeri S, Monaco S, Yildiz I, Silvi S, Raymo FM, et al. Structural and Size Effects on the Spectroscopic and Redox Properties of CdSe Nanocrystals in Solution: The Role of Defect States. ChemPhysChem. 2011;12(12):2280-2288.

46. Ghosh B, Shirahata N. Colloidal silicon quantum dots: synthesis and luminescence tuning from the near-UV to the near-IR range. Science and Technology of Advanced Materials. 2014;15(1):014207.

47. Flores NM, Pal U, Galeazzia R, Sandoval A. Effects of morphology, surface area, and defect content on the photocatalytic dye degradation performance of $\mathrm{ZnO}$ nanostructures. RSC Advances. 2014;4(77):41099-41110.

48. Unni C, Philipb D, Smitha SL, Nissamudeen KM, Gopchandran KG. Aqueous synthesis and characterization of CdS, CdS: $\mathrm{Zn}^{2+}$ and CdS: $\mathrm{Cu}^{2+}$ quantum dots. Spectrochimica Acta Part A: Molecular and Biomolecular Spectroscopy. 2009;72(4):827-832.

49. Wang Y, Lu JP, Tong ZF. Rapid synthesis of CdSe nanocrystals in aqueous solution at room temperature. Bulletin of Materials Science. 2010;33(5):543-546.

50. Liu YF, Yu JS. Selective synthesis of CdTe and high luminescence $\mathrm{CdTe} / \mathrm{CdS}$ quantum dots: The effect of ligands". Journal of Colloid and Interface Science. 2009;333(2):690-698.

51. Zhang JL, Han BX, Liu DX, Chen J, Liu ZM, Mu TC, et al. Effects of ultrasound on the microenvironment in reverse micelles and synthesis of nanorods and nanofibers. Physical Chemistry Chemical Physics. 2004;6(9):2391-2395. 